\title{
A Limit Theorem for Products of Free Unitary Operators
}

\author{
Vladislav Kargin ${ }^{* \dagger}$
}

\begin{abstract}
This paper establishes necessary and sufficient conditions for the products of freely independent unitary operators to converge in distribution to the uniform law on the unit circle.
\end{abstract}

AMS Subject Classification: 46L53, 46L54, 60F05

KeYwords: Free Probability, Free Multiplicative Convolution, Unitary Op-

erators, Limit Theorem

\section{Introduction}

Suppose $X$ is a unitary $n$-by- $n$ matrix. Then $X$ has $n$ eigenvalues, which are all located on the unit circle. If we give each eigenvalue a weight of $n^{-1}$, then we can think about the distribution of these eigenvalues as a probability distribution supported on $n$ points of the unit circle. More generally, if $X$ is a unitary operator in a finite von Neumann algebra, then we can define a spectral probability distribution of $X$, which is supported on the unit circle (see, e.g., Section 1.1 in Hiai and Petz [2000]).

If we have several unitary operators $X_{1}, \ldots, X_{n}$, then it is natural to ask about the spectral distribution of their product. In general, we cannot determine this distribution without more information about relations among operators $X_{1}, \ldots, X_{n}$. However, if $X_{1}, \ldots, X_{n}$ are infinite-dimensional and, in a certain sense, in a general position relative to each other, then the spectral distribution of their product is computable. The idea of a general position was

\footnotetext{
${ }^{*}$ Courant Institute of Mathematical Sciences, 251 Mercer Street, New York, NY 10012; kargin@cims.nyu.edu

†The author would like to express his gratitude to Diana Bloom for her help with editing, and to Professor Raghu Varadhan for useful discussions.
} 
formalized by Voiculescu in his concept of freeness of operators (see Voiculescu 1983] Voiculescu [1986], and a textbook by Hiai and Petz [2000]). If operators $X_{1}, \ldots, X_{n}$ are free and unitary and their spectral probability distributions are $\mu_{1}, \ldots, \mu_{n}$, respectively, then the distribution of their product is determined uniquely. This distribution is called the free multiplicative convolution of measures $\mu_{1}, \ldots, \mu_{n}$ and denoted as $\mu_{1} \otimes \ldots \otimes \mu_{n}$.

What can we say about the asymptotic behavior of $\mu^{(n)}=: \mu_{1} \otimes \ldots \otimes \mu_{n}$, as $n$ increases to infinity? In particular, what are necessary and sufficient conditions on $\mu_{i}$ that ensure that $\mu^{(n)}$ converges to the uniform distribution on the unit circle?

To answer this question, let us define the expectation with respect to the measure $\mu_{i}$. This is a functional that maps functions analytic in a neighborhood of the unit circle to complex numbers:

$$
E_{\mu_{i}} f=: \int_{|\xi|=1} f(\xi) d \mu_{i}(\xi) .
$$

If unitary operator $X_{i}$ has the spectral probability distribution $\mu_{i}$, then we will also write:

$$
\operatorname{Ef}\left(X_{i}\right)=: E_{\mu_{i}} f .
$$

In particular, $E X_{i}$ denotes $\int_{|\xi|=1} \xi d \mu_{i}(\xi)$. Then the answer is given by the following theorem:

Theorem 1 Suppose $\left\{X_{i}\right\}_{i=1}^{\infty}$ are free unitary operators with spectral measures $\mu_{i}$. The measures $\mu^{(n)}$ of the products $\Pi_{n}=: X_{1} \ldots X_{n}$ converge to the uniform measure on the unit circle if and only if at least one of the following situations holds:

(i) There exist two indices $i \neq j$ such that $E X_{i}=E X_{j}=0$;

(ii) There exists exactly one index $i$ such that $E X_{i}=0$, and $\prod_{k=i+1}^{n} E X_{k} \rightarrow 0$ as $n \rightarrow \infty$;

(iii) There exists exactly one index $i$ such that $X_{i}$ has the uniform distribution;

(iv) $E X_{k} \neq 0$ for all $k$, and $\prod_{k=1}^{n} E X_{k} \rightarrow 0$ as $n \rightarrow \infty$.

In other words, convergence of $\mu^{(n)}$ to the uniform law implies that $\prod_{k=1}^{n} E X_{k} \rightarrow$ 0 , and the only case when the reverse implication fails is when $E X_{i}=0$ for exactly one $X_{i}$, the measure $\mu_{i}$ is not uniform, and $\prod_{k=i+1}^{n} E X_{k} \nrightarrow 0$ as $n \rightarrow \infty$. Note that cases (ii) and (iii) above are not exclusive. It may happen that both $\mu_{i}$ is uniform and $\prod_{k=i+1}^{n} E X_{k} \rightarrow 0$ as $n \rightarrow \infty$. In this case, both (ii) and (iii) hold, and $\mu^{(n)}$ converges to the uniform law.

This theorem can be thought of as a limit theorem about free multiplicative convolutions of measures on the unit circle. There is some literature about traditional multiplicative convolutions of measures on the unit circle, or more generally, about convolutions of measures on compact groups. For the unit 
circle, this investigation was started by Levy [1939]. Then it was continued by Kawada and Itô 1940], who studied compact groups, and Dvoretzky and Wolfowitz [1951] and Vorobev [1954], who both considered the case of commutative finite groups. These researchers found an important necessary condition for convergence of convolutions to the uniform law. This condition requires that there should be no normal subgroup such that the convolved measures are supported entirely in an equivalence class relative to this subgroup. This condition is sufficient if summands are identically distributed. If they are not, then there are some sufficient and necessary conditions, which are especially useful if the group is cyclic. A textbook presentation with further references can be found in Grenander [1963.

Recent investigations of convolutions on groups are mostly concerned with the speed of convergence of convolved measures to the uniform law. For a description of progress in this direction, the reader can consult surveys in Diaconis [1988] and Saloff-Coste [2004].

It turns out that free convolutions converge to the uniform law under much weaker conditions than usual convolutions. As an example, consider the distributions that are concentrated on -1 and +1 . Let measure $\mu_{k}$ put the weight $p_{k}$ on +1 . Then usual convolutions remain concentrated on -1 and +1 , and therefore they have no chance to converge to the uniform distribution on the unit circle. In contrast, we will show that free convolutions do converge to the uniform law, provided that either $\prod_{k=k_{0}}^{n}\left(2 p_{k}-1\right) \rightarrow 0$ for arbitrarily large $k_{0}$, or there exist two indices $i$ and $j$ such that $p_{i}=p_{j}=1 / 2$.

The rest of the paper is organized as follows. Section 2 provides the necessary background. In Section 3 we outline the proof. Section 4 derives some auxiliary results that will be used in the proof. Section 5 proves the main result (Theorem 1). Section 6 derives the key estimate used in the proof. And Section 7 concludes.

\section{Definitions and Background}

Definition $2 A$ non-commutative probability space is a pair $(\mathcal{A}, E)$, where $\mathcal{A}$ is a unital $C^{*}$-algebra of bounded linear operators acting on a complex separable Hilbert space and $E$ is a linear functional from $\mathcal{A}$ to complex numbers. The operators from algebra $\mathcal{A}$ are called non-commutative random variables, or simply random variables, and the functional $E$ is called the expectation.

The linear functional $E$ is assumed to satisfy the following properties (in addition to linearity): i) $E(I)=1$; ii) $E\left(A^{*}\right)=E(A)$; iii) $E\left(A A^{*}\right) \geq 0$; iv) $E\left(A A^{*}\right)=0$ implies $A=0$; and v) if $A_{n} \rightarrow A$ then $E\left(A_{n}\right) \rightarrow E(A)$, where convergence of operators is in norm. 
If $P(d \lambda)$ is the spectral resolution associated with a unitary operator $A$, then we can define a measure $\mu(d \lambda)=E(P(d \lambda))$. It is easy to check that $\mu$ is a probability measure supported on the unit circle. We call this measure, $\mu$, the spectral probability measure associated with operator $A$ and expectation E.

The most important concept in free probability theory is that of free independence of non-commuting random variables. Let a set of r.v. $A_{1}, \ldots, A_{n}$ be given. With each of them we can associate an algebra $\mathcal{A}_{i}$, which is generated by $A_{i}$; that is, it is the closure of all polynomials in variables $A_{i}$ and $A_{i}^{*}$. Let $\bar{A}_{i}$ denote an arbitrary element of algebra $\mathcal{A}_{i}$.

Definition 3 The algebras $\mathcal{A}_{1}, \ldots, \mathcal{A}_{n}$ (and variables $A_{1}, \ldots, A_{n}$ that generate them) are said to be freely independent or free, if the following condition holds:

$$
\varphi\left(\bar{A}_{i(1)} \ldots \bar{A}_{i(m)}\right)=0
$$

provided that $\varphi\left(\bar{A}_{i(s)}\right)=0$ and $i(s+1) \neq i(s)$.

For more information about non-commutative probability spaces and free operators we refer the reader to Sections $2.2-2.5$ in the book by Voiculescu et al. [1992].

We will use two results regarding the free operators, which we cite without proofs. The first one is formula (2.2.3) on page 44 in Hiai and Petz [2000].

Proposition 4 Let $\mathcal{A}_{1}, \ldots, \mathcal{A}_{m}$ be free sub-algebras of $\mathcal{A}$, and let $A_{1}, \ldots, A_{n}$ be a sequence of random variables, $A_{k} \in \mathcal{A}_{i(k)}$ such that $i(k) \neq i(k+1)$. Then

$E\left(A_{1} \ldots A_{n}\right)=\sum_{r=1}^{n} \sum_{1 \leq k_{1}<\ldots<k_{r} \leq n}(-1)^{r-1} E\left(A_{k_{1}}\right) \ldots E\left(A_{k_{r}}\right) E\left(A_{1} \ldots \widehat{A}_{k_{1}} \ldots \widehat{A}_{k_{r}} \ldots A_{n}\right)$,

where ${ }^{\wedge}$ denotes terms that are omitted.

Remark: Note that on the right-hand side the expectations are taken of the products that have no more than $n-1$ terms. So a recursive application of this formula reduces computation of $E\left(X_{1} \ldots X_{n}\right)$ to a polynomial in the moments of the individual variables.

The second result is the Voiculescu multiplication theorem. To formulate it we need some additional definitions.

Define the $\psi$-function of a bounded random variable $X$ as

$$
\psi_{X}(z)=: \sum_{k=1}^{\infty} E\left(X^{k}\right) z^{k}
$$

If $X$ is unitary operator with the spectral measure $\mu$, then we can write: 


$$
\psi_{\mu}(z)=\int_{|\xi|=1} \frac{1}{1-\xi z} d \mu(\xi)-1 .
$$

It is very useful to note that the $\psi$-function is related to the Poisson transform of the measure $\mu$. Indeed, since

$$
\operatorname{Re} \frac{1}{1-\xi z}=\frac{1-r \cos (\omega-\theta)}{1-2 r \cos (\omega-\theta)+r^{2}}
$$

where $\xi=e^{-i \theta}$ and $z=r e^{i \omega}$, therefore,

$$
\operatorname{Re} \frac{1}{1-\xi z}=\frac{1}{2}+\pi P(r, \omega-\theta)
$$

where $P(r, \theta)$ is the Poisson kernel:

$$
P(r, \theta)=\frac{1}{2 \pi} \frac{1-r^{2}}{1-2 r \cos \theta+r^{2}} .
$$

Recall that the Poisson transform of a measure $\mu$ supported on the unit circle is defined as

$$
U_{\mu}(z)=: \int_{-\pi}^{\pi} P(r, \omega-\theta) d \mu(\theta),
$$

where $z=r e^{i \omega}$. (Here we have identified measures on the unit circle and on the interval $[-\pi, \pi): \mu(d \theta)=\mu\{\xi:|\xi|=1$ and $\arg \xi \in d \theta\})$. Hence,

$$
U_{\mu}(z)=\frac{1}{\pi} \operatorname{Re} \psi(z)+\frac{1}{2 \pi} .
$$

Let $\psi_{X}^{-1}(u)$ denote the functional inverse of $\psi_{X}(z)$ in a neighborhood of $z=0$, where $\psi_{X}(z)$ is as defined in (2). (This inversion is possible provided that $E(X) \neq 0$.) Define also

$$
S_{X}(u)=\frac{u+1}{u} \psi_{X}^{-1}(u) .
$$

Theorem 5 [Voiculescu] Suppose $X$ and $Y$ are bounded free random variables. Suppose also that $E(X) \neq 0$ and $E(Y) \neq 0$. Then

$$
S_{X Y}(z)=S_{X}(z) S_{Y}(z) \text {. }
$$

The original proof can be found in Voiculescu [1987]. A simpler proof was given by Haagerup [1997]. Using this theorem, it is possible to compute the free convolution of two measures, $\mu_{1}$ and $\mu_{2}$. First, we can compute their $\psi$ functions, $\psi_{\mu_{1}}$ and $\psi_{\mu_{2}}$. Then we invert them and obtain the $S$-functions, $S_{\mu_{1}}$ and $S_{\mu_{2}}$. Their product is the $S$-function of the free convolution, $S_{\mu_{1}} \otimes_{\mu_{2}}$, and we can compute $\psi_{\mu_{1} \bowtie \mu_{2}}$ by inversion. This determines the Poisson transform of $\mu_{1} \otimes \mu_{2}$, from which we can determine the measure itself. (For the one-to-one relation of Poisson transforms and corresponding measures, see Theorem I.3.1 on page 15 and a comment on page 20 in Garnett [1981].) 


\section{Outline of the Proof}

Let $\Pi_{n}$ denote the partial products: $\Pi_{n}=X_{1} \ldots X_{n}$. We denote $E\left(X_{i}\right)$ as $a_{i}$, and $E\left(\Pi_{n}\right)$ as $a_{(n)}$. First, note that it is enough to consider the case when all $a_{i}$ are real and non-negative. Indeed, for an arbitrary sequence of real constants $\theta_{n}$, the sequence of operators $e^{i \theta_{n}} \Pi_{n}$ converges in distribution to the uniform law if and only if the sequence $\Pi_{n}$ converges in distribution to the uniform law. (Indeed, if, say, $\Pi_{n}$ does not converge in distribution to the uniform law, then we can find an integer $k$ such that $\left|\int_{0}^{2 \pi} e^{i k \theta} \mu_{(n)}(d \theta)\right| \nrightarrow 0$, where $\mu_{(n)}$ denotes the measure of $\Pi_{n}$. But then $\left|\int_{0}^{2 \pi} e^{i k \theta} e^{i \theta_{n}} \mu_{(n)}(d \theta)\right|=\left|\int_{0}^{2 \pi} e^{i k \theta} \mu_{(n)}(d \theta)\right| \nrightarrow 0$, and this implies $e^{i \theta_{n}} \Pi_{n}$ does not converge in distribution to the uniform law.) Therefore if $a_{i}=E\left(X_{i}\right)$ is not real and positive, then we can replace $X_{i}$ with $e^{-i \arg a_{i}} X_{i}$ without affecting the convergence of $\Pi_{n}$.

We divide the analysis into the following cases:

Case I $a_{(n)} \nrightarrow 0$.

Case II $a_{(n)} \rightarrow 0$, and there are at least two indices, $i$ and $j$, such that $a_{i}=a_{j}=0$.

Case III $a_{(n)} \rightarrow 0$, and for all $i, a_{i}>0$.

Subcase III.1 $\liminf a_{i}=0$.

Subcase III.2 $\liminf a_{i}=\underline{a}>0$.

Case IV $a_{(n)} \rightarrow 0$, and there exists exactly one index $i$, such that $a_{i}=0$.

We will show that without loss of generality we can assume in this case that $a_{1}=0$, and $a_{k}>0$ for all $k>1$.

Subcase IV.1 $X_{1}$ has the uniform distribution.

Subcase IV.2 $X_{1}$ does not have the uniform distribution, and $\prod_{k=2}^{n} a_{n} \rightarrow$ 0 as $n \rightarrow \infty$.

Subcase IV.3 $X_{1}$ does not have the uniform distribution, and $\prod_{k=2}^{n} a_{n} \nrightarrow$ 0 as $n \rightarrow \infty$.

We will show that $\Pi_{n}$ does not converge to the uniform law if and only if either Case I or Case IV.3 holds.

\section{Auxiliary Lemmas}

We will need to perform functional inversions. A useful tool for doing this is Lagrange's formula.

Lemma 6 [Lagrange's inversion formula] Suppose that (i) $f$ is a function of a complex variable $z$, which is analytic in a neighborhood of $z=0$, (ii) $f(0)=0$, and (iii) $f^{\prime}(0)=a \neq 0$. Then the functional inverse of $f(z)$ is well defined in a neighborhood of 0 , and the Taylor series of the inverse is given by the following 
formula:

$$
f^{-1}(u)=\frac{u}{a}+\sum_{k=2}^{\infty}\left[\frac{1}{k} \operatorname{res}_{z=0} \frac{1}{f(z)^{k}}\right] u^{k}
$$

where $\operatorname{res}_{z=0}$ denotes the Cauchy residue at 0 . In addition,

$$
f^{-1}(u)=\frac{u}{a}+\sum_{k=2}^{\infty}\left[\frac{1}{2 \pi i k} \oint_{\gamma} \frac{d z}{f(z)^{k}}\right] u^{k}
$$

where $\gamma$ is a circle around 0 , inside which $f$ has only one zero.

For a proof see Section 7.32 in Whittaker and Watson [1927].

We will also use the lemmas below:

Lemma 7 Suppose $A$ and $B$ are free unitary operators, $|E(A)| \leq a$ and $|E(B)| \leq$ $b$. Then for all integer $k \geq 1$,

$$
\left|E\left[(A B)^{k}\right]\right| \leq M_{k} \max (a, b)
$$

for certain constants $M_{k}$, which depend only on $k$.

Proof: If we expand $E\left[(A B)^{k}\right]$ using Proposition 4, then we can observe that each term in the expansion contains either $E(A)$ or $E(B)$ as a separate multiple. The remaining multiples in this term are $\leq 1$ in absolute value; therefore, we can bound each term by $\max (a, b)$. The number of terms in this expansion is bounded by a constant, $M_{k}$. Therefore, $\left|E\left[(A B)^{k}\right]\right|$ is bounded by $M_{k} \max (a, b)$. QED.

In the following lemmas we use the fact that the sequence of probability measures $\mu_{i}$, supported on the unit circle, converges to the uniform law if and only if all their moments converge to 0 , that is, iff for each $k \geq 1, \int_{|\xi|=1} \xi^{k} d \mu_{i}(\xi) \rightarrow 0$ as $i \rightarrow \infty$. For completeness we give a proof of this result.

Let us define $c_{k}^{(i)}=: \int_{|\xi|=1} \xi^{k} d \mu_{i}(\xi)$. Note that for a fixed $i, c_{k}^{(i)}$ are coefficients in the Taylor series of $\psi_{i}(z)$, i.e., the $\psi$-function of the measure $\mu_{i}$.

Lemma 8 Let $\mu_{i}$ be a sequence of measures supported on the unit circle. If for each $k$ the coefficients $c_{k}^{(i)} \rightarrow 0$ as $i \rightarrow \infty$, then $\psi_{i}(z) \rightarrow 0$ uniformly on compact subsets of the open unit disc.

Proof: Let $\Omega$ be a compact subset of the open unit disc, and let $\Omega \subset D_{r}$, where $D_{r}$ denotes a closed disc with the radius $r<1$. Fix an $\varepsilon \in(0,1)$. Then we can find such a $k_{0}$ that

$$
\left|\sum_{k=k_{0}}^{\infty} c_{k}^{(j)} z^{k}\right|<\varepsilon / 2
$$


for all $z \in D_{r}$ and all $j$. Indeed, $\left|c_{k}^{(j)}\right| \leq 1$, and therefore,

$$
\left|\sum_{k=k_{0}}^{\infty} c_{k}^{(j)} z^{k}\right| \leq \frac{r^{k_{0}}}{1-r}
$$

so we can take $k_{0}$ to be any integer greater than or equal to $\log (\varepsilon(1-r) / 2) / \log r$.

Given $k_{0}$, we choose a $j_{0}$ so large that for all $j>j_{0}$ and all $k<k_{0}$, we have $\left|c_{k}^{(j)}\right|<\varepsilon /\left(2 k_{0}\right)$. This is possible because by assumption for each $k$ coefficients $c_{k}^{(j)}$ converge to zero as $j \rightarrow \infty$, and we consider only a fixed finite number of possible $k$.

Consequently,

$$
\left|\sum_{k=1}^{k_{0}-1} c_{k}^{(j)} z^{k}\right| \leq \sum_{k=1}^{k_{0}-1}\left|c_{k}^{(j)}\right|<\varepsilon / 2
$$

for every $j>j_{0}$ and all $z \in D_{r}$. Therefore,

$$
\left|\sum_{k=1}^{\infty} c_{k}^{(j)} z^{k}\right|<\varepsilon
$$

for every $j>j_{0}$ and all $z \in D_{r}$. Therefore, $\psi_{j}(z) \rightarrow 0$ uniformly on $D_{r}$, and therefore on $\Omega$. Since $\Omega$ was arbitrary, we have proved that $\psi_{j}(z) \rightarrow 0$ uniformly on compact subsets of the unit disc. QED.

The fact that $\psi_{j}(z) \rightarrow 0$ implies that the Poisson transforms of measures $\mu_{j}$ converge to $\frac{1}{2 \pi}$, and therefore $\mu_{j} \rightarrow \nu$, where $\nu$ is the uniform measure on the unit disc. Indeed, we only need to invoke the following result:

Proposition 9 If Poisson transforms $U_{\mu_{j}}(z) \rightarrow 1 /(2 \pi)$ uniformly on compact subsets of the unit disc, then $\mu_{j}$ weakly converges to $\nu$, where $\nu$ is the uniform probability measure on the unit circle.

Proof: This proposition directly follows from Theorem I.3.1 on page 15 in Garnett [1981], adapted to the case of measures on the unit disc. QED.

Lemma 10 Suppose $\left\{A_{n}\right\}_{n=1}^{\infty}$ is a sequence of unitary operators that converges in distribution to the uniform law. Let $\left\{B_{n}\right\}_{n=1}^{\infty}$ be another sequence of unitary operators, and let the operator $B_{n}$ be free of the operator $A_{n}$ for every $n$. Then the sequence of products $B_{n} A_{n}$ converges in distribution to the uniform law. Also, the sequence $A_{n} B_{n}$ converges to the uniform law.

Proof: Let $a_{k}^{(n)}=: E\left(\left(A_{n}\right)^{k}\right)$. By assumption, for each fixed $k$, the moment $a_{k}^{(n)} \rightarrow 0$ as $n \rightarrow \infty$. If we represent $E\left(\left(B_{n} A_{n}\right)^{k}\right)$ as a polynomial in individual moments of $B_{n}$ and $A_{n}$, then all terms of this polynomial contain at least one of 
the moments $a_{i}^{(n)}, i \leq k$, which are perhaps multiplied by some other moments. All of these other moments are less than 1 in absolute value. Therefore, we can write the following estimate:

$$
\left|E\left(\left(B_{n} A_{n}\right)^{k}\right)\right| \leq M_{k}^{\prime} \max _{i \leq k}\left\{a_{i}^{(n)}\right\},
$$

where $M_{k}^{\prime}$ is the number of terms in the polynomial. If $k$ is fixed and $n$ is growing, then the assumption that $A_{n}$ converges in distribution to the uniform law implies that $\max _{i \leq k}\left\{a_{i}^{(n)}\right\}$ converges to zero. Therefore, all moments of $B_{n} A_{n}$ converge to zero as $n \rightarrow \infty$, and therefore, by Lemma 8 and Proposition 9, the sequence $B_{n} A_{n}$ converges in distribution to the uniform law. A similar argument proves that $A_{n} B_{n}$ converges in distribution to the uniform law. QED.

Lemma 11 Suppose that $B$ is a unitary operator, $\left\{A_{n}\right\}$ is a sequence of unitary operators, $B$ is free from each of $A_{n}, E(B) \neq 0$, and the sequence $A_{n}$ does not converge to uniform law. Then the sequence of products $B A_{n}$ does not converge to the uniform law.

Proof: The condition that the sequence $A_{n}$ does not converge to the uniform law means that for some fixed $k$ the sequence of $k$-th moments of $A_{n}$ does not converge to zero as $n \rightarrow \infty$. Let $k$ be the smallest of these indices. By selecting a subsequence we can assume that $\left|E\left(A_{n}^{k}\right)\right|>\alpha>0$ for all $n$. Consider $E\left(\left(B A_{n}\right)^{k}\right)$ :

$$
E\left(\left(B A_{n}\right)^{k}\right)=[E(B)]^{k} E\left(A_{n}^{k}\right)+\ldots,
$$

The number of the terms captured by ... is finite and depends only on $k$. Each of these terms includes at least one of $E\left(A_{n}^{i}\right)$ where $i<k$, and other multipliers in this term are less than 1 in absolute value. Therefore, each of these terms converges to zero. Hence, for any $\varepsilon>0$, there exist such $N$ that for all $n>N$, the sum of the terms captured by $\ldots$ is less than $\varepsilon$ in absolute value. Take $\varepsilon=|E(B)|^{k} \alpha / 2$. Then for $n>N$, we have:

$$
\left|E\left(\left(B A_{n}\right)^{k}\right)\right| \geq|E(B)|^{k} \alpha / 2 .
$$

Therefore, the sequence of products $B A_{n}$ does not converge to the uniform law. QED.

Lemma 12 Suppose that $B$ is a unitary random variable, $\left\{A_{n}\right\}$ is a sequence of unitary random variables, $B$ is free from each of $A_{n}, B$ is not uniform, and the sequence of expectations $E\left(A_{n}\right)$ does not converge to zero. Then the sequence of products $B A_{n}$ does not converge to the uniform law. 
Proof: By selecting a subsequence we can assume that $\left|E\left(A_{n}\right)\right|>\alpha>0$ for all $n$. The assumption that $B$ is not uniform means that for some $k \geq 1$, $E\left(B^{k}\right) \neq 0$. Let $k$ be the smallest of such $k$. Consider $E\left(\left(B A_{n}\right)^{k}\right)$ :

$$
E\left(\left(B A_{n}\right)^{k}\right)=\left[E\left(A_{n}\right)\right]^{k} E\left(B^{k}\right)+\ldots,
$$

Each of the terms in $\ldots$ includes one of $E\left(B^{i}\right)$ where $i<k$. Therefore, all terms in ... are zero. Hence,

$$
\left|E\left(\left(B A_{n}\right)^{k}\right)\right|=\left|\left[E\left(A_{n}\right)\right]^{k} E\left(B^{k}\right)\right|>\alpha^{k}\left|E\left(B^{k}\right)\right| .
$$

Therefore, the sequence of products $B A_{n}$ does not converge to the uniform law. QED.

\section{Analysis}

We use the following notation: $\psi_{i}$ and $S_{i}$ denote $\psi$ - and $S$-functions for variables $X_{i}$ (and measures $\mu_{i}$ ), and $\psi_{(n)}$ and $S_{(n)}$ denote these functions for variables $\Pi_{n}$ (and measures $\left.\mu^{(n)}\right)$.

Case I: $a_{(n)} \nrightarrow 0$.

Since $E\left(\Pi_{n}\right)=a_{(n)}$, therefore, if $a_{(n)} \nrightarrow 0$, then $E\left(\Pi_{n}\right) \nrightarrow 0$. Hence, $\Pi_{n}$ cannot converge to the uniform measure on the unit circle.

Case II $a_{(n)} \rightarrow 0$, and there are at least two indices $i$ and $j$ such that $a_{i}=a_{j}=0$.

Assume without loss of generality that $j>i$. Consider $\Pi_{n}$ with $n \geq j$ and define $X=: X_{1} \ldots X_{i}$ and $Y=: X_{i+1} \ldots X_{n}$. Then $\Pi_{n}=X Y$, and $E(Y)=$ $E(X)=0$. Using Lemma 7 , we obtain that $\left|E\left[\left(\Pi_{n}\right)^{k}\right]\right|=0$ for every $k>0$. Therefore, the $\psi$-function of $\Pi_{n}$ is zero, and $\Pi_{n}$ has the uniform distribution on the unit circle.

Case III $a_{(n)} \rightarrow 0$, and for all $i, a_{i}>0$.

Subcase III.1 $\liminf a_{i}=0$.

In this case we can find a subsequence $a_{n_{i}}$ that monotonically converges to zero.

Now, consider $\Pi_{j}$, where $j \in\left[n_{i}, n_{i+1}\right)$. Then we can write $\Pi_{j}=X Y$, where $X=X_{1} \ldots X_{n_{i}-1}$, and $Y=X_{n_{i}} \ldots X_{j}$. Then $E X \leq a_{n_{i-1}}$ and $E Y \leq a_{n_{i}} \leq$ $a_{n_{i-1}}$.

Applying Lemma 7 we get

$$
\left|E\left(\Pi_{j}^{k}\right)\right| \leq M_{k} a_{n_{i-1}} .
$$

This implies that for a fixed $k,\left|E\left(\Pi_{j}^{k}\right)\right|$ approaches zero as $j \rightarrow \infty$. By Lemma 8 and Proposition 9, this establishes that $\Pi_{j}$ converges to the uniform law. 
Case III $a_{(n)} \rightarrow 0$, and for all $i, a_{i}>0$

Subcase III.2 $\liminf a_{i}=\underline{a}>0$.

Let us choose such an $a$ that $0<a<\underline{a}$. Starting from some $j_{0}, a_{j} \in(a, 1)$. Let $\widetilde{\Pi}_{n}=X_{j_{0}} \ldots X_{n+j_{0}-1}$. Then, by Lemmas 10 and 11, $\widetilde{\Pi}_{n}$ converges to the uniform law if and only if $\Pi_{n}$ converges to the uniform law Hence, without loss of generality we can restrict our attention to the case when $a_{k} \in(a, 1)$ for all $k$.

Lemma 13 Suppose $1 \geq a_{k}>0$ for all $k$, and let $\alpha_{i}=: 1-a_{i}$. Then $\prod_{i=1}^{n} a_{i} \rightarrow$ 0 if and only if $\sum_{i=1}^{n} \alpha_{i} \rightarrow \infty$.

This is a standard result. For a proof see Section 2.7 in Whittaker and Watson [1927].

Since $\log \left(1-\alpha_{i}\right) \leq-\alpha_{i}$, we also have the following estimate, which we will find useful later.

$$
\prod_{i=1}^{n} a_{i} \leq \exp \left(-\sum_{i=1}^{n} \alpha_{i}\right) .
$$

To prove convergence to the uniform law, we have to establish that for every $k>0$ the coefficient $c_{k}^{(n)}$ in the Taylor expansion of function $\psi_{(n)}(z)$ approaches zero as $n \rightarrow \infty$. We know from Lemma 6 that

$$
k c_{k}^{(n)}=\operatorname{res}_{z=0} \frac{1}{\left[\psi_{(n)}^{-1}(z)\right]^{k}} ;
$$

therefore, our main task is to estimate this residual. This is the same as estimating the coefficient before the term $z^{k-1}$ in the Taylor expansion of

$$
f(z)=\left[\frac{z}{\psi_{(n)}^{-1}(z)}\right]^{k} .
$$

We will approach this problem by using the Cauchy inequality (see Section 5.23 in Whittaker and Watson [1927]). Applied to the coefficient before $z^{k-1}$ in the Taylor expansion of $f(z)$, this inequality says that

$$
\left|k c_{k}^{(n)}\right| \leq \frac{M(r)}{r^{k-1}}
$$

where $r>0$ is such that $f(z)$ is analytic inside $|z|=r$, and

$$
M(r)=: \max _{|z|=r}|f(z)| .
$$

It is easy to check that the constant in the Taylor expansion of $z / \psi_{(n)}^{-1}(z)$ is $a_{(n)}$. So $M(0)=a_{(n)}$, which approaches zero as $n \rightarrow \infty$. The main question is how large we can take $r$, so that $M(r)$ remains relatively small. In other words, we want to minimize the right-hand side of (5) by a suitable choice of $r$. 
Proposition 14 Suppose that $E X_{i}=a_{i}>a$ for each $i$ and that $a_{(n)}=$ : $\prod_{i=1}^{n} a_{i} \rightarrow 0$. Let $\alpha_{i}=1-a_{i}$. Then for all sufficiently large $n$, the following inequality holds:

$$
\left|c_{k}^{(n)}\right| \leq\left(\frac{C}{a^{2}}\right)^{k}\left[\left(\sum_{i=1}^{n} \alpha_{i}\right) \exp \left(-\sum_{i=1}^{n} \alpha_{i}\right)\right]^{k},
$$

where $C=2^{17}$.

Proof: The main tool in the proof is the following proposition:

Proposition 15 Suppose that $\alpha_{i}=: 1-a_{i}<1-a$ for each $i$, and that $z$ and $n$ are such that

$$
|z| \leq \frac{a^{2}}{6684} \min \left\{1,\left(\sum_{i=1}^{n} \alpha_{i}\right)^{-1}\right\} .
$$

Then,

$$
\left|\frac{z}{\psi_{(n)}^{-1}(z)}\right|^{k} \leq\left(2 e^{2}\right)^{k}\left(\prod_{i=1}^{n} a_{i}\right)^{k} .
$$

We will prove this proposition in the next section and assume for now that it holds.

Let $n_{0}$ be so large that $\sum_{i=1}^{n_{0}} \alpha_{i}>1$. (We can find such $n_{0}$ because by Lemma 13. $\sum_{i=1}^{n} \alpha_{i} \rightarrow \infty$ as $n \rightarrow \infty$.) In particular, this implies that $\sum_{i=1}^{n} \alpha_{i}>1$ for every $n \geq n_{0}$. Define $r_{n}=: a^{2}\left(\sum_{i=1}^{n} \alpha_{i}\right)^{-1} / 6684$. Then, using Proposition 15 and formulas (5) and (4), we get:

$$
\begin{aligned}
\left|k c_{k}^{(n)}\right| & \leq\left(2 e^{2}\right)^{k}\left(\prod_{i=1}^{n} a_{i}\right)^{k}\left(\frac{6684}{a^{2}} \sum_{i=1}^{n} \alpha_{i}\right)^{k-1} \\
& \leq\left[\frac{2^{17}}{a^{2}}\left(\sum_{i=1}^{n} \alpha_{i}\right) \exp \left(-\sum_{i=1}^{n} \alpha_{i}\right)\right]^{k}
\end{aligned}
$$

provided that $n \geq n_{0}$. QED.

Using Lemma 13, we get the following Corollary:

Corollary 16 If the assumptions of Proposition 14 hold, then for each $k$, the coefficient $c_{k}^{(n)} \rightarrow 0$ as $n \rightarrow \infty$.

This Corollary shows that in Case III.2 the product $\Pi_{n}$ converges to the uniform law.

Case IV $a_{(n)} \rightarrow 0$, and there exists exactly one index $i$, such that $a_{i}=0$.

First, we want to show that without loss of generality we can assume in this case that $a_{1}=0$, and $a_{k}>0$ for all $k>1$. Indeed, suppose $a_{i}=0$ for $i>1$, 
and $a_{j}>0$ for $j<i$. Let $X=X_{1} \ldots X_{i-1}$ and let $\widetilde{\Pi}_{n}=X_{i} \ldots X_{i+n-1}$. Then $E(X) \neq 0$, and using Lemmas 10 and 11, we conclude that $\Pi_{n}$ converges to the uniform law if and only if $\widetilde{\Pi}_{n}$ converges to the uniform law.

\section{Subcase IV.1 $X_{1}$ has the uniform distribution.}

In this case all moments of $X_{1}$ are zero, i.e., $E\left(X_{1}^{k}\right)=0$ for all $k>0$, and Proposition 4 implies that all moments of $\Pi_{n}$ are zero. Therefore, $\Pi_{n}$ is uniform for all $n$.

Subcase IV.2 $X_{1}$ does not have the uniform distribution, and $\prod_{k=2}^{n} a_{n} \rightarrow$ 0 as $n \rightarrow \infty$.

By Case III, the product $X_{2} \ldots X_{n}$ converges to the uniform law, and using Lemma 10, we conclude that $\Pi_{n}$ also converges to the uniform law.

Subcase IV.3 $X_{1}$ does not have the uniform distribution and $\prod_{k=2}^{n} a_{n} \nrightarrow$ 0 as $n \rightarrow \infty$.

Applying Lemma 12 to $B=X_{1}$ and $A=X_{2} \ldots X_{n}$, we conclude that $\Pi_{n}$ does not converge to the uniform law.

\section{Proof of Proposition 15}

Let

$$
f(z)=:\left(\frac{z}{\psi_{(n)}^{-1}(z)}\right)^{k} .
$$

Using Theorem 5 , we can write this function as follows:

$$
f(z)=\left(\frac{z^{n}}{(1+z)^{n-1}} \prod_{i=1}^{n} \frac{1}{\psi_{i}^{-1}(z)}\right)^{k} .
$$

We want to estimate $|f(z)|$ for all sufficiently small $z$. We start with some auxiliary estimates, which will later allow us to estimate $\psi_{i}(z)$, and then $\psi_{i}^{-1}(z)$ for small $z$.

Lemma 17 Suppose $\mu$ is a probability measure on $[-\pi, \pi)$ such that

$$
\left|\int_{-\pi}^{\pi}\left(e^{i \theta}-1\right) d \mu(\theta)\right| \leq \alpha .
$$

Then, i)

$$
\int_{-\pi}^{\pi} \theta^{2} d \mu(\theta) \leq \frac{\pi^{2}}{2} \alpha<5 \alpha
$$

ii)

$$
\left|\int_{-\pi}^{\pi} \theta d \mu(\theta)\right| \leq\left(1+\frac{\pi^{3}}{12}\right) \alpha<4 \alpha, \text { and }
$$


iii) if $k>2$, then

$$
\int_{-\pi}^{\pi}|\theta|^{k} d \mu(\theta) \leq \frac{\pi^{k}}{2} \alpha
$$

Proof: Condition (7) implies that

$$
\int_{-\pi}^{\pi}(1-\cos (\theta)) d \mu(\theta) \leq \alpha
$$

and that

$$
\left|\int_{-\pi}^{\pi} \sin (\theta) d \mu(\theta)\right| \leq \alpha
$$

Since $1-\cos \theta \geq\left(2 / \pi^{2}\right) \theta^{2}$, from the first of these inequalities we infer that:

$$
\int_{-\pi}^{\pi} \theta^{2} d \mu(\theta) \leq\left(\pi^{2} / 2\right) \alpha
$$

which proves claim i) of the lemma.

Next, note that $|\sin \theta-\theta| \leq|\theta|^{3} / 6$, and that

$$
\frac{1}{6} \int_{-\pi}^{\pi}|\theta|^{3} d \mu(\theta) \leq \frac{\pi}{6} \int_{-\pi}^{\pi} \theta^{2} d \mu(\theta) \leq \frac{\pi^{3}}{12} \alpha .
$$

Therefore,

$$
\begin{aligned}
\left|\int_{-\pi}^{\pi} \theta d \mu(\theta)\right| & \leq\left|\int_{-\pi}^{\pi} \sin (\theta) d \mu(\theta)\right|+\left|\int_{-\pi}^{\pi}(\theta-\sin (\theta)) d \mu(\theta)\right| \\
& \leq \alpha+\frac{\pi^{3}}{12} \alpha .
\end{aligned}
$$

This proves claim ii) of the lemma.

For claim iii), note that

$$
\int_{-\pi}^{\pi}|\theta|^{k} d \mu(\theta) \leq \pi^{k-2} \int_{-\pi}^{\pi}|\theta|^{2} d \mu(\theta) \leq \frac{\pi^{k}}{2} \alpha .
$$

QED.

Lemma 18 Suppose Condition (7) holds, and $k$ is a positive integer. Then

$$
\left|\int_{-\pi}^{\pi}\left(e^{i k \theta}-1\right) d \mu(\theta)\right| \leq 7 k^{3} \alpha
$$

Proof: First, remark that $1-\cos (k \theta) \leq(k \theta)^{2} / 2$ and therefore

$$
\begin{aligned}
\left|\int_{-\pi}^{\pi}(\cos k \theta-1) d \mu(\theta)\right| & \leq \frac{k^{2}}{2} \int_{-\pi}^{\pi} \theta^{2} d \mu(\theta) \\
& \leq \frac{\pi^{2} k^{2}}{4} \alpha
\end{aligned}
$$


Next, we will use $|\sin (k \theta)-k \theta| \leq(k|\theta|)^{3} / 6$ and write

$$
\begin{aligned}
\left|\int_{-\pi}^{\pi} \sin (k \theta) d \mu(\theta)\right| & \leq\left|\int_{-\pi}^{\pi} k \theta d \mu(\theta)\right|+\left|\frac{1}{6} \int_{-\pi}^{\pi}(k|\theta|)^{3} d \mu(\theta)\right| \\
& \leq k\left(1+\frac{\pi^{3}}{12}\right) \alpha+\frac{1}{6} k^{3} \frac{\pi^{3}}{2} \alpha \\
& \leq k^{3}\left(1+\frac{\pi^{3}}{6}\right) \alpha .
\end{aligned}
$$

Consequently,

$$
\begin{aligned}
\left|\int_{-\pi}^{\pi}\left(e^{i k \theta}-1\right) d \mu(\theta)\right| & \leq \alpha \sqrt{\frac{\pi^{4} k^{4}}{16}+k^{6}\left(1+\frac{\pi^{3}}{6}\right)^{2}} \\
& \leq 7 k^{3} \alpha .
\end{aligned}
$$

QED.

Lemma 19 Let $X$ be unitary and $E X=a>0$. If $|z| \leq 1 / 2$ and $1-a \leq \alpha$, then

$$
\left|\psi_{X}(z)-\frac{a z}{1-z}\right| \leq 716 \alpha|z|^{2}
$$

Proof: We can write:

$$
\psi_{X}(z)-\frac{a z}{1-z}=\sum_{k=2}^{\infty}\left(E\left(X^{k}\right)-a\right) z^{k}
$$

Therefore, using Lemma 18, we estimate:

$$
\begin{aligned}
\left|\psi_{X}(z)-\frac{a z}{1-z}\right| & \leq \sum_{k=2}^{\infty}\left(\left|E\left(X^{k}\right)-1\right|+|1-a|\right) z^{k} \\
& \leq 7 \alpha|z|^{2} \sum_{k=0}^{\infty}\left[(k+2)^{3}+1 / 7\right]|z|^{k} \\
& \leq 716 \alpha|z|^{2} .
\end{aligned}
$$

(Note that 716 is the exact value of the sum $7 \sum_{k=0}^{\infty}\left[(k+2)^{3}+1 / 7\right] 2^{-k}$.) QED.

To derive a similar estimate for $\psi_{X}^{-1}(z)$, we need a couple of preliminary lemmas.

Lemma 20 Suppose $X$ is unitary and $E X=a>0$. Then the function $\psi_{X}(z)$ has only one zero $(z=0)$ in the area $|z|<a / 3$. If $|z|=a / 3$, then $\left|\psi_{X}(z)\right| \geq$ $a^{2} / 6$. 
Proof: Write the following estimate:

$$
\begin{aligned}
\left|\psi_{X}(z)-a z\right| & =\left|\sum_{k=2}^{\infty} E\left(X^{k}\right) z^{k}\right| \\
& \leq|z| \sum_{k=1}^{\infty}|z|^{k}=\frac{|z|}{1-|z|}|z|<\frac{a}{2}|z|,
\end{aligned}
$$

if $|z|<a / 3$. By Rouché's theorem, $\psi_{X}(z)$ has only one zero in $|z|<a / 3$. The second claim also follows immediately from this estimate. QED.

Lemma 21 Suppose $X$ is unitary and $E X=a>0$. Then the function $\psi_{X}^{-1}(z)$ is analytical for $|z|<a^{2} / 6$. If $|z| \leq a^{2} / 12$, then

$$
\left|\psi_{X}^{-1}(z)\right| \leq \frac{2}{a}|z|
$$

Proof: Using Lagrange's formula, we can write

$$
\psi_{X}^{-1}(z)=\frac{z}{a}+\sum_{k=2}^{\infty} c_{k} z^{k}
$$

where

$$
c_{k}=\frac{1}{2 \pi i} \frac{1}{k} \oint_{\gamma} \frac{d u}{\left[\psi_{X}(u)\right]^{k}} .
$$

By the previous lemma, we can use the circle with the center at 0 and radius $a / 3$ as $\gamma$, and then we can estimate $c_{k}$ as follows:

$$
\left|c_{k}\right| \leq \frac{a / 3}{k\left(a^{2} / 6\right)^{k}}=\frac{2}{k a}\left(\frac{6}{a^{2}}\right)^{k-1} .
$$

It follows that the power series for $\psi_{X}^{-1}(z)$ converges in $|z|<a^{2} / 6$. If $|z|<a^{2} / 12$, then we can estimate $\psi_{X}^{-1}(z)$ :

$$
\begin{aligned}
\left|\psi_{X}^{-1}(z)\right| & \leq \frac{|z|}{a}\left(1+a \sum_{k=2}^{\infty}\left|c_{k}\right||z|^{k-1}\right) \\
& \leq \frac{|z|}{a}\left(1+\sum_{k=2}^{\infty}\left(\frac{6}{a^{2}}\right)^{k-1}|z|^{k-1}\right) \\
& \leq \frac{|z|}{a} \frac{1}{1-\frac{6}{a^{2}}|z|} \leq \frac{2}{a}|z|
\end{aligned}
$$

where in the second line we used inequality (8). QED.

Lemma 22 Let $X$ be unitary and $E X=a>0$. If $|z| \leq a^{2} / 12$, and $\alpha \geq 1-a$, then

$$
\left|\frac{\psi_{X}^{-1}(z)}{z /(a+z)}-1\right| \leq \frac{3342}{a^{2}} \alpha|z| .
$$


Proof: First of all, by Lemma 21

$$
\left|\psi_{X}^{-1}(z)\right| \leq \frac{2}{a}|z|
$$

for $|z| \leq a^{2} / 12$.

Now we use the functional equation for $\psi_{X}^{-1}(z)$ :

$$
\psi_{X}\left(\psi_{X}^{-1}(z)\right)=z .
$$

If $|z| \leq a^{2} / 12$, then $\left|\psi_{X}^{-1}(z)\right| \leq 2|z| / a \leq a / 6<1 / 2$ and we can apply Lemma 19 to get:

$$
\begin{aligned}
\left|z-\frac{a \psi_{X}^{-1}(z)}{1-\psi_{X}^{-1}(z)}\right| & \leq 716 \alpha\left|\psi_{X}^{-1}(z)\right|^{2} \\
& \leq 716 \alpha \frac{4}{a^{2}}|z|^{2}=\frac{2864 \alpha}{a^{2}}|z|^{2} .
\end{aligned}
$$

Next, we write this as

$$
\begin{aligned}
\left|z-(a+z) \psi_{X}^{-1}(z)\right| & \leq\left|1-\psi_{X}^{-1}(z)\right| \frac{2864 \alpha}{a^{2}}|z|^{2} \\
& \leq \frac{7}{6} \frac{2864 \alpha}{a^{2}}|z|^{2}<\frac{3342 \alpha}{a^{2}}|z|^{2} .
\end{aligned}
$$

(In the second inequality we used the fact that $\left|\psi_{X}^{-1}(z)\right| \leq 1 / 6$ if $|z| \leq a^{2} / 12$.) It follows that

$$
\left|\frac{\psi_{X}^{-1}(z)}{z /(a+z)}-1\right| \leq \frac{3342}{a^{2}} \alpha|z| .
$$

QED.

Lemma 23 Let $E X_{i}=a_{i}$ and assume that for each $i$, it is true that $a_{i} \geq a$. Assume also that $|z| \leq a^{2} / 3342$ and let $\alpha_{i}=: 1-a_{i}$. Then

$$
\left|\prod_{i=1}^{n} \frac{1}{\psi_{i}^{-1}(z)}\right| \leq \frac{\prod_{i=1}^{n} a_{i}}{|z|^{n}} \prod_{i=1}^{n} \frac{1}{1-c_{i}|z|}\left|\prod_{i=1}^{n}\left(1+\frac{z}{a_{i}}\right)\right|,
$$

where $c_{i}=3342 \alpha_{i} / a_{i}^{2}$.

Proof: From Lemma 22 we infer that

$$
\left|\psi_{i}^{-1}(z)\right| \geq\left|\frac{z}{a_{i}} \frac{1}{1+z / a_{i}}\right|\left(1-\frac{3342 \alpha_{i}}{a_{i}^{2}}|z|\right) .
$$

Multiplying these inequalities together and inverting both sides, we get the desired result. QED. 
Lemma 24 Under the assumptions of the previous lemma, the following inequality holds:

$$
|f(z)| \leq\left(|1+z| \prod_{i=1}^{n} a_{i} \prod_{i=1}^{n} \frac{1}{1-c_{i}|z|}\left|\prod_{i=1}^{n} \frac{1+z / a_{i}}{1+z}\right|\right)^{k},
$$

where $c_{i}=3342 \alpha_{i} / a_{i}^{2}$.

Proof: The claim of this lemma is a direct consequence of Lemma 23 and equality (6). QED.

We will estimate terms in the product on the right-hand side of (9) one by one.

Lemma 25 Suppose that $\alpha_{i}=: 1-a_{i}<1-a$ for each $i$, and that

$$
|z| \leq \frac{a^{2}}{6684} \min \left\{1,\left(\sum_{i=1}^{n} \alpha_{i}\right)^{-1}\right\} \text {. }
$$

Then

$$
\left|\prod_{i=1}^{n} \frac{1+z / a_{i}}{1+z}\right| \leq e
$$

Proof: We write:

$$
\left|\prod_{i=1}^{n} \frac{1+z / a_{i}}{1+z}\right|=\exp \left(\operatorname{Re} \sum_{i=1}^{n} \log \left(1+\frac{\alpha_{i}}{a_{i}} \frac{z}{1+z}\right)\right) .
$$

Recall that $\operatorname{Re} \log (1+u) \leq|u|$ if $|u|<1$. Under our assumption about $|z|$, it is true that

$$
\left|\frac{\alpha_{i}}{a_{i}} \frac{z}{1+z}\right|<1 .
$$

Therefore we can write:

$$
\begin{aligned}
\left|\prod_{i=1}^{n} \frac{1+z / a_{i}}{1+z}\right| & \leq \exp \left(\left|\frac{z}{1+z}\right| \sum_{i=1}^{n} \frac{\alpha_{i}}{a_{i}}\right) \\
& \leq \exp \left(\frac{2}{a}|z| \sum \alpha_{i}\right) \\
& \leq e .
\end{aligned}
$$

QED.

Lemma 26 Suppose that $\alpha_{i}=: 1-a_{i}<1-a$ for each $i$, and that

$$
|z| \leq \frac{a^{2}}{6684} \min \left\{1,\left(\sum_{i=1}^{n} \alpha_{i}\right)^{-1}\right\} \text {. }
$$


Then,

$$
\prod_{i=1}^{n} \frac{1}{1-c_{i}|z|} \leq e
$$

where $c_{i}=3342 \alpha_{i} / a_{i}^{2}$.

Proof: We use the inequality $\log (1-u) \geq-2 u$, which is valid for $u \in$ $(0,1 / 2)$, and write:

$$
\begin{aligned}
\prod_{i=1}^{n} \frac{1}{1-c_{i}|z|} & =\exp \left(-\sum_{i=1}^{n} \log \left(1-c_{i}|z|\right)\right) \\
& \leq \exp \left(2|z| \sum_{i=1}^{n} c_{i}\right) \\
& \leq \exp \left[\frac{6684}{a^{2}}\left(\sum_{i=1}^{n} \alpha_{i}\right)|z|\right] \\
& \leq e .
\end{aligned}
$$

QED.

Finally, note that if $|z| \leq a^{2} / 6684$, then $|1+z| \leq 2$. Collecting all the pieces, we obtain that if

$$
|z| \leq \frac{a^{2}}{6684} \min \left\{1,\left(\sum_{i=1}^{n} \alpha_{i}\right)^{-1}\right\}
$$

then:

$$
|f(z)| \leq\left(2 e^{2}\right)^{k}\left(\prod_{i=1}^{n} a_{i}\right)^{k} .
$$

This completes the proof of Proposition 15 .

\section{Conclusion}

We have derived sufficient and necessary conditions for the product of free unitary operators to converge in distribution to the uniform law. If essential convergence denotes the situation when the partial products continue to converge even after an arbitrary finite number of terms are removed, then the necessary and sufficient condition for essential convergence is that the products $\prod_{i=k_{0}}^{n} E X_{i}$ converges to zero for all $k_{0}$, that is, that the products of expectations essentially converge to zero. Essential convergence implies convergence. In addition, nonessential convergence can occur when there is either a term that has the uniform distribution, or there are two terms that have zero expectation. In the latter case convergence occurs because the product of these two terms has the uniform distribution. 


\section{References}

P. Diaconis. Group Representations in Probability and Statistics. Institute of Mathematical Statistics, 1988. Volume 11 in Lecture Notes - Monograph Series of IMS.

A. Dvoretzky and J. Wolfowitz. Sums of random integers reduced modulo $\mathrm{m}$. Duke Mathematical Journal, 18:501-507, 1951.

J. B. Garnett. Bounded Analytic Functions, volume 96 of Pure and Applied Mathematics. Academic Press, New York, 1 edition, 1981.

U. Grenander. Probabilities on Algebraic Structures. John Wiley and Sons Inc., 1963.

U. Haagerup. On Voiculescus $R$ - and $S$-transforms for free non-commuting random variables. In D.-V. Voiculescu, editor, Free Probability Theory, volume 12 of Fields Institute Communications, pages 127-148. American Mathematical Society, 1997.

F. Hiai and D. Petz. The Semicircle Law, Free Random Variables And Entropy, volume 77 of Mathematical Surveys and Monographs. American Mathematical Society, 1 edition, 2000.

Y. Kawada and K. Itô. On the probability distribution on a compact group. Proceedings of Physical and Mathematical Society of Japan, 22:977-998, 1940.

P. Levy. L'addition des variables aleatoires definies sur une circonference. In D. Dugue, editor, Oeuvres de Paul Levy, volume 3, pages 452-492. GauthierVillars, (1976), 1939.

L. Saloff-Coste. Random walks on finite groups. In H. Kesten, editor, Probability on Discrete Structures, pages 263-346. 2004. Volume 110 in Encyclopaedia of Mathematical Sciences.

D. Voiculescu. Symmetries of some reduced free product $\mathrm{C}^{*}$-algebras. In Lecture Notes in Mathematics, volume 1132, pages 556-588. Springer-Verlag, New York, 1983.

D. Voiculescu. Addition of certain non-commuting random variables. Journal of Functional Analysis, 66:323-346, 1986.

D. Voiculescu. Multiplication of certain non-commuting random variables. Journal of Operator Theory, 18:223-235, 1987.

D. Voiculescu, K. Dykema, and A. Nica. Free Random Variables. A.M.S. Providence, RI, 1992. CRM Monograph series, No.1. 
N. N. Vorobev. The addition of independent random variables on finite groups. Matematicheskii Sbornik, 34:89-126, 1954. In Russian.

E. T. Whittaker and G. N. Watson. A Course of Modern Analysis. Cambridge University Press, 4 edition, 1927. 